\title{
Chemical and Thermal History of the Intracluster Medium
}

\author{
Hans Böhringer \\ Max-Planck-Institut für extraterrestrische Physik, Garching, Germany
}

\begin{abstract}
Clusters of galaxies can be seen as giant astrophysical laboratories enclosing matter in a large enough volume, so that the matter composition can be taken as representing the composition of our Universe. $\mathrm{X}$-ray observations allow a very precise investigation of the physical properties of the intracluster plasma allowing us to probe the cluster structure, determine its total mass, and measure the baryon fraction in clusters and in the Universe as a whole. We can determine the abundance of heavy elements from $\mathrm{O}$ to $\mathrm{Ni}$ which originate from supernova explosions and draw from this important conclusions on the history of star formation in the cluster galaxy population. From the entropy structure of the intracluster medium we obtain constraints on the energy release during early star bursts. With the observational capabilities of the X-ray observatories XMM-Newton and Chandra this field of research is rapidly evolving. In particular, first detailed observations of the intracluster medium of the Virgo cluster around M87 have provided new insights. The present contribution gives an account of the current implications of the intracluster medium observations, but more importantly illustrates the prospects of this research for the coming years.
\end{abstract}

\section{Introduction}

Clusters of galaxies, the largest well defined objects, are the largest well characterized astrophysical laboratories at our disposal, except for the Universe as a whole. The clusters masses range from less than $10^{14}$ to several $10^{15} \mathrm{M}_{\odot}$. This mass range is extended down to about several $10^{12} \mathrm{M}_{\odot}$ by galaxy groups which can be considered as small scale versions of clusters. Hundreds to thousands of cluster galaxies are making up merely a few percent of the total cluster mass while more mass is in the gaseous intracluster medium (ICM). The dominant fraction is Dark Matter. Being formed through gravitational collapse where gravity acts on all forms of matter equally, galaxy clusters contain to a good approximation a composition of matter well representative of the Universe as a whole (e.g. White et al. 1993).

In this contribution I will focus on the information we can gain on galaxy evolution from the study of the chemical composition and the thermodynamic structure of the ICM. One of the astronomically most interesting epochs in the history of our Universe was that of the most intense star and galaxy formation at redshift between 2 and 4 (e.g. Madau et al. 1998). The traces of what happened then can be found in the ICM today: i) we observe that the ICM is enriched by 
metals which must have been synthesized by supernovae in the cluster galaxies mostly in these early star formation epochs; ii) we further observe in the entropy structure of groups and clusters of galaxies the effect of an early heating of the intergalactic medium by energy release in the star formation epochs.

The ICM is a hot plasma with temperatures of several tens of million degrees which has its thermal radiation maximum in the soft X-ray regime. It is a fortunate coincidence that it is the same wavelength region in which we can use the current technology of imaging X-ray telescopes. Therefore we know most about the ICM through X-ray astronomy. We have furthermore two new satellite Xray observatories, ESA's XMM-Newton mission and NASA's Chandra mission, with greatly improved spectral and imaging capabilities, which are providing overwhelming new insight into the physics of the ICM. In this contribution I will use a varying scaling of physical parameters by the Hubble constant (depending on the sources) quoted which will be labelled by the scaling parameter $h$, e.g. $h_{70}=H_{0} / 70 \mathrm{~km} \mathrm{~s}^{-1} \mathrm{Mpc}^{-1}$.

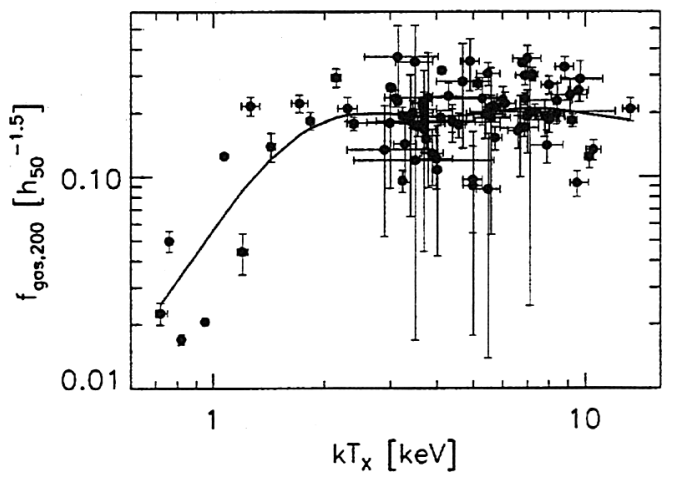

Figure 1. Gas mass fraction in units of $h_{50}^{-1.5}$ for 106 of the brightest clusters of galaxies detected by ROSAT in X-rays (from Reiprich 2001).

\section{The mass fraction of the intergalactic gas}

A prime characteristic of the matter budget is the fraction of baryonic matter with respect to the Dark Matter. The gas mass as well as the total mass can be determined from the gas density and temperature distribution of the ICM (assuming hydrostatic equilibrium) which is in turn determined from X-ray imaging and spectroscopy. Fig. 1 shows the gas mass fractions determined for a sample of clusters observed with ROSAT and ASCA (Reiprich 2001, Reiprich \& Böhringer 2002). The data scatter around a value of $18-20 \%$ (for $h=0.5$; or $12 \%$ for $h=0.7)$. The large error bars in the mass are due to uncertainties in the temperature and temperature profiles. The gas mass fraction seems to be quite constant for massive clusters but decreases towards the galaxy groups. With the capability of spatially resolved spectroscopy with XMM Newton and Chandra the precision of such measurements greatly improves. One of the best 

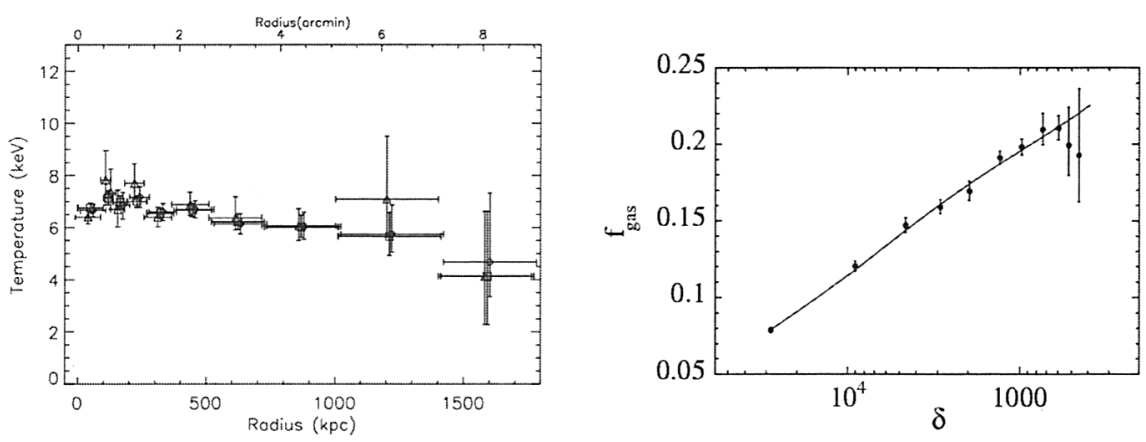

Figure 2. Right panel: Temperature profile of the cluster A1413 determined from XMM-Newton spectroscopic observations by Pratt \& Arnaud (2002). This temperature profile stretches out to the largest radii in a cluster analysis reached so far, to 0.6 of the virial radius. Left panel: Gas mass fraction in A1413 from Pratt \& Arnaud (2002).

recent examples is the XMM study of the galaxy cluster A1413 shown in Fig. 2 (Pratt \& Arnaud 2002). Here the gas mass fraction has been determined out to a radius close to the assumed virial radius of the cluster. The resulting gas mass fraction is $0.2( \pm 0.02) h_{50}^{-1.5}$. Similar results with a more limited radial range have been obtained with the CHANDRA observatory (e.g. Allen et al. 2001, Ettori et al. 2002).

Assuming that most of the unseen matter is non-baryonic, we can determine the baryon fraction of the total cluster mass by summing the observed gas and galaxy mass (the latter making up about $2-3 \%$ of the total mass) finding values around $12 \% h_{70}^{-1.5}$. Taking this fraction as representative of the Universe together with the matter density of the Universe as provided by the cosmological "concordance" model (e.g. Turner 2002) we get $\Omega_{b} h^{2}=0.0191$. This is consistent with the value from nucleosynthesis for the observed primordial deuterium to hydrogen ratio (e.g. O'Meara et al. 2001) giving $\Omega_{b} h^{2}=0.020$ and the analysis of microwave background observations providing $\Omega_{b} h^{2}=0.0224$ (for $h=0.7$ and $\Omega_{m}=0.3$ ). This general consistency is also a confirmation of the mass and gas mass fraction determination in clusters.

\section{Observations of the metallicity of the intracluster medium before XMM-Newton}

The first studies of the metal abundance distribution with ASCA and BeppoSAX observations showed a central increase of the $\mathrm{Fe}$ abundance in clusters with so-called cooling flows (e.g. Matsumoto et al. 1996, Fukazawa et al. 1998, Finoguenov et al. 2000, 2001), while clusters without a dense core and without a central $\mathrm{cD}$ galaxy display flat iron profiles as shown in Fig. 3 (De Grandi \& Molendi 2001). These results lead to the tentative implication that the central Fe abundance increase is connected to recent iron production in the central early type galaxies. The principle sources of the heavy elements are the two types 

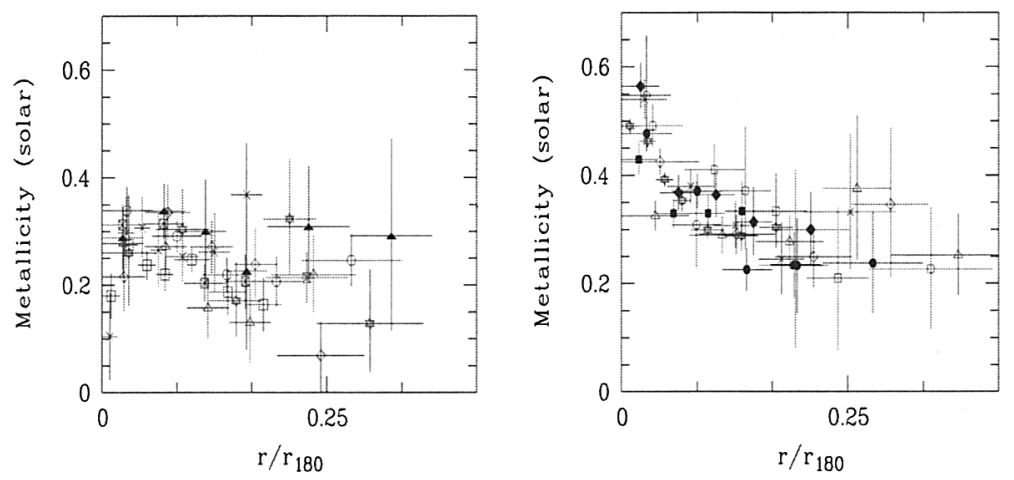

Figure 3. Iron abundance profiles of non-cooling flow clusters (left) and cooling flow clusters (right) as a function of the scaled radius expressed in units of the radius at a mean mass overdensity of 180 over the critical density of the Universe (De Grandi \& Molendi 2001).

of supernovae, type II (core collapse supernovae) and type Ia (thermonuclear exploding white dwarfs). Only the supernovae of the type Ia have been observed in the early type galaxies. Therefore the increased abundance of iron in the cluster centers must come from the enrichment by SN type Ia. This can be tested by looking at the relative abundances of different elements produced in different ratios by SN Ia and SN II. Using ASCA results Fukazawa et al. (1998) and Finoguenov et al. (2000, 2001a) showed a clear trend for an increase in the Si to Fe ratio with radius, implying that SN II with relative high Si yields are dominating in the non-central cluster regions.

\section{Metal abundance determination in the X-ray halo of M87 with XMM-Newton}

The giant elliptical galaxy, M87, in the center of the northern part of the Virgo cluster is the nearest X-ray luminous galaxy cluster center, providing the highest signal-to-noise for X-ray spectral analysis and the best angular resolution for the study of the dense central ICM. In addition the temperature of the X-ray halo of M87 is with values in the range from 1 to $3 \mathrm{keV}$ in a regime where the spectra are very rich in emission lines. For its importance M87 was one of the verification targets of the XMM-Newton mission.

To determine element abundances the temperature structure of the ICM has to be known with high precision. In particular, the M87 halo was previously believed to harbour a classical cooling flow, proposed to display a complex multi-temperature structure with a wide range of temperatures (e.g. Nulsen 1986, Thomas et al. 1987, Fabian 1994) which can easily lead to ambiguous abundance results (e.g. Buote 1999). Therefore the first analysis was a careful determination of the temperature structure of the M87 halo (Matsushita et al. 2002). It turns out that the classical cooling flow picture does not apply (Fig. 4, Böhringer et al. 2001, 2002, Moldendi et al. 2001, Molendi 2002) and that 
the gas is very close have a single temperature phase locally, but shows a monotonic decrease in temperature towards the center (Matsushita et al. 2002) which greatly facilitates the data interpretation.
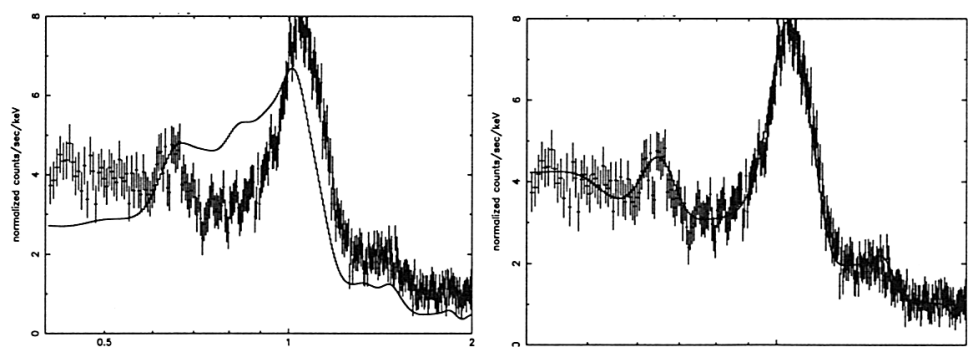

Figure 4. The observed Fe L line feature in the X-ray halo of M87 (around a radius of 2 arcmin) compared to a single temperature (right) and a classical cooling flow model (left; Böhringer et al. 2002).

Abundance profiles in M87 determined (for deprojected spectra) for the elements $\mathrm{O}, \mathrm{Si}, \mathrm{S}$, and Fe are shown in Fig. 5 (Matsushita et al. 2003a). We clearly see a different behavior of the different elements. While $\mathrm{O}$ shows a relatively flat profile, the profiles of $\mathrm{Si}$ and $\mathrm{Fe}$ decrease significantly with radius (in approximately the same way) and S seems to fall off even more steeply. This is partly what we could have expected: oxygen which is produced essentially only by SN type II at early epochs and which should be well mixed shows the more even distribution. The strong increase in $\mathrm{Fe}$ and $\mathrm{Si}$ is then due to the enrichment by SN Ia. It comes somewhat as a surprise, however, that the profiles of $\mathrm{Fe}$ and $\mathrm{Si}$ are practically running in parallel. We should have expected a stronger increase of $\mathrm{Fe}$ compared to $\mathrm{Si}$, because in the classical models the SN type Ia which are responsible for the central increase of the abundance profiles, should produce predominantly iron and less Si.

To investigate this further the behavior of all the elements was studied by Finoguenov et al. (2001b) in two radial bins (1 to $3 \mathrm{arcmin}$ and 8 to $16 \operatorname{arcmin}$ ) in M87. The result was deconvolved into the contributions of the two different types of supernovae under the assumption that the central abundance increase is entirely due to SN type Ia. One can then subtract the outer abundance pattern from the inner one to obtain the SN Ia yields. Alternatively one can use oxygen as a tracer of the contribution of SN type II and subtract from the inner abundances a weighted outer abundance pattern such that the $\mathrm{O}$ abundance is zero. (see Finoguenov et al. 2002 for details). The results of this procedure are shown in Fig. 6.

These deconvolved abundance patterns are compared to theoretical models in the Figure. For the SN type Ia yields models by Nomoto et al. (1997, see also Iwamoto et al. 1999) are used for the comparison. The model with the lowest yields for the $\alpha$-elements compared to the Fe group elements is the classical Nomoto et al. (1984) W7 model of a fast SN Ia explosion which has been widely used for chemical evolution models. The relative yields inferred from the present observations show much larger $\alpha$-element yields more in line with the slow deflagration-detonation models featuring a less complete burning of the $\alpha$ - 

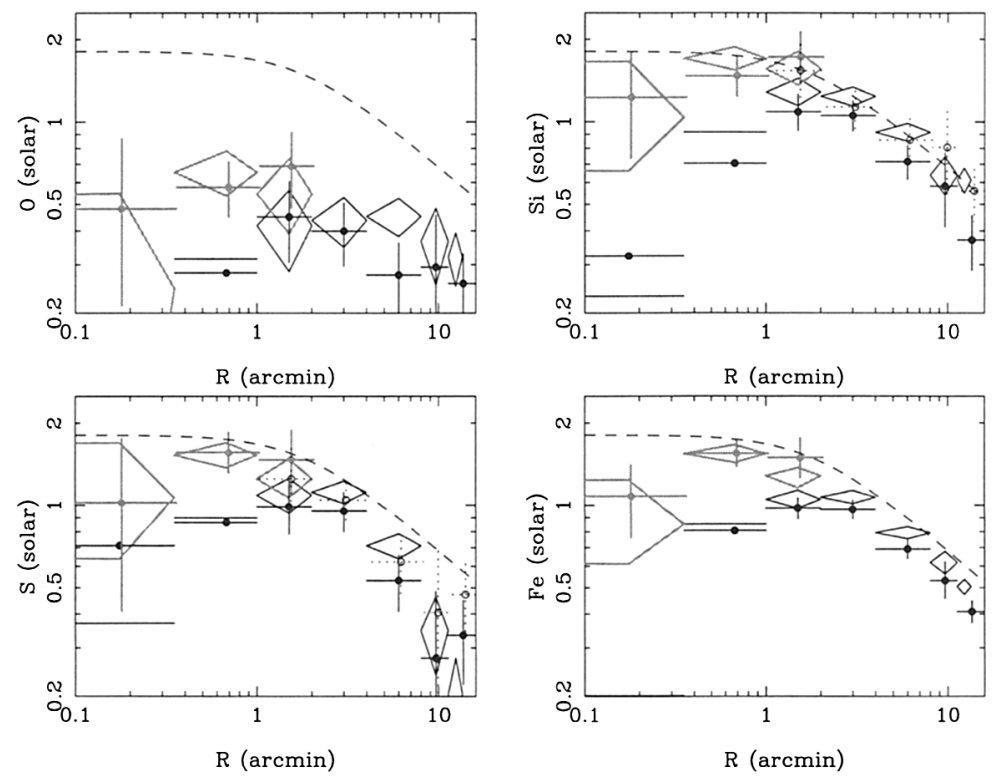

Figure 5. $\mathrm{O}, \mathrm{Si}, \mathrm{S}$ and $\mathrm{Fe}$ abundance profiles in $\mathrm{M} 87$ (Matsushita et al. 2003a). The grey symbols came from a two-temperature model (grey symbols) for the central region where the disturbance of the central AGN may not be perfectly subtracted. These results are more reliable than the simple one-temperature treatment (black symbols). The data are obtained from the two XMM detectors EPN (crosses) and EMOS (diamonds).

elements. One also notes a second order result that the two patters of SN type Ia for the inner and outer region do not perfectly agree, but the outer region seems to feature relatively lower yields for the $\alpha$-elements. This may be an indication of a secular variation of the nature of SN Ia supernovae, with a smaller yield (less complete burning and less bright light curves) for Fe group elements for a younger stellar population and a relatively higher yield of $\alpha$-elements in the very old stellar populations of elliptical galaxies, like M87. This is in line with the statistics that faint SN Ia are preferentially detected in early type galaxies (Ivanov et al. 2000). This was investigated in more detail by Matsushita et al. (2003a, 2003b) in M87 and Centaurus confirming this picture. An analysis of the enrichment times involved in the production of the central metal abundance enhancement is described in Böhringer et al. (2003b) where it is found that the enrichment time in the very inner zone of M87 (inside the scale radius of about $10 \mathrm{kpc}$ ) is about $2-3$ Gyrs while in the enrichment time integrated out to $50 \mathrm{kpc}$ has to be of the order of 10 Gyrs. This enrichment age difference that roughly applies to the two radial zones shown in Fig. 6 can quite well be related to a significant difference in the stellar population and enrichment histories of the inner and outer region of the M87 halo. 

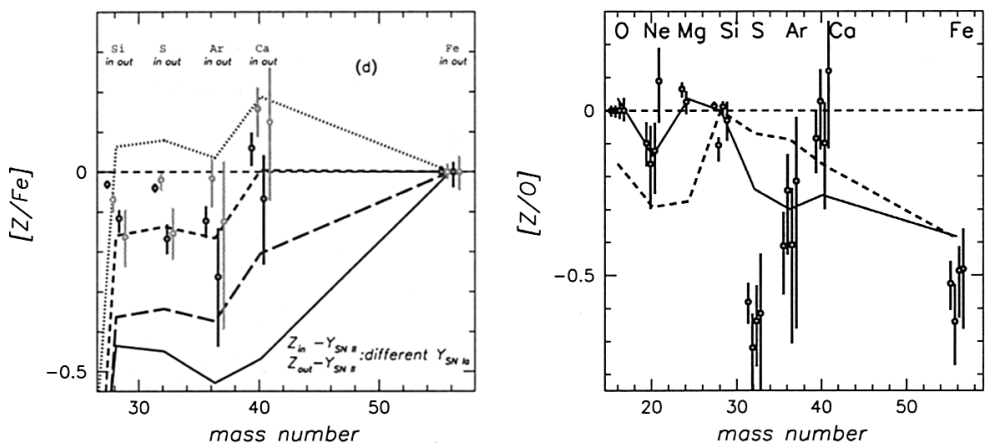

Figure 6. The contribution of SN type Ia (left) and SN II (right) to the abundance pattern in M87 (Finoguenov et al. 2002). Also shown is a comparison with nuclear synthesis model calculations: left panel: the lowest model curve is the classical SN Ia model of a fast explosion by Nomoto et al. 1984, the other three curves are slow deflagrationdetonation models with the higher curves having decreasing deflagration speeds and less complete burning (Nomoto et al. 1997). Right panel: Shows a comparison with SN II models by Nomoto et al. 1997 (upper curve) and Woosley and Weaver (lower curve).

\section{Entropy structure of galaxy groups and clusters}

The formation of dark matter halos from primordial overdensities is a self similar process. The structure of the groups and clusters of galaxies is essentially determined by the potential shape of the dark matter halos in which they are embedded. Therefore many of the properties of clusters are well characterized by self-similar scaling relations, like the mass - ICM temperature relation which is predicted to have a logarithmic slope of 1.5 which is approximately observed for massive clusters (e.g. Finoguenov et al. 2001b). A closer comparison of groups and clusters shows, however, that the predicted scaling relations are not always very well observed - the temperature - X-ray luminosity (bolometric luminosity) relation is a good example where the observed slope is steeper than predicted (e.g. Navarro et al. 1996).

The possible reason for this is revealed in the diagnostics pioneered by Ponman et al. (1999). Shown in Fig. 7 the entropy at a radius of $0.1 r_{200}$ (an inner radius avoiding cooling flow regions scaled to the virial radius $r_{200}$ of the cluster) as a function of system temperature. The hot gas temperature and the entropy is in general the result of shock heating of the gas when the cluster is subsequently assembled by a series of substructure mergers. The shock strength should thereby be proportional to the depth of the gravitational potential in which the accreted matter settles which is itself proportional to the temperature. Thus we expect that the gas entropy (expressed here with the specific definition of $S=T / n^{2 / 3}$ ) is proportional to the shock strength and thus to the temperature. The shallower slope observed is indicative of an extra source of heat which affects specifically the low temperature systems. Such an effect is easily explained if the heat input is proportional to the gas mass, that is a 

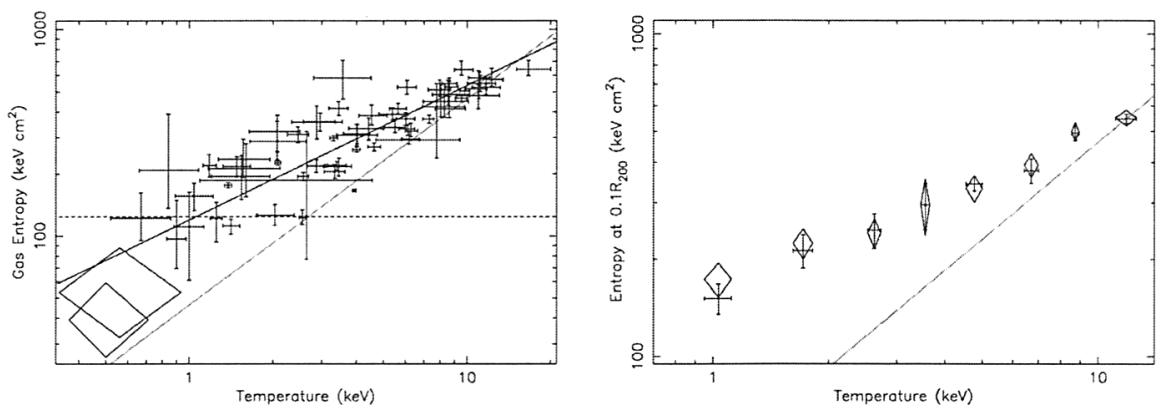

Figure 7. Entropy at a radius of $0.1 r_{200}$ as a function of ICM temperature for groups and clusters of galaxies (left) including two galaxies (diamonds). Same data grouped into bins of 8 objects excluding the galaxies (right). The solid line shows the best fit and the dotted line the self-similar slope of 1 (Ponman et al. 2003)

mechanism that provides a source of fixed specific thermal energy. A heat input proportional to the stellar population mass that is heating from star formation would fullfil this requirement. The extra entropy in the central regions amounts to typical values of 100 to $200 \mathrm{keV} \mathrm{cm}{ }^{2}$.

One obvious source of a fixed amount of specific energy is the energy released by those supernovae which also contribute to the enrichment of the intracluster medium. From the above considerations on the metal enrichment of the intracluster medium we can also calculate the energy that is released by the same supernovae. Assuming that not much energy is dissipated locally in the galaxies where the supernovae explode but that most of it is transported out by a galactic wind, one finds that about 0.5 to $0.75 \mathrm{keV}$ per proton is available (see e.g. also Finoguenov et al. 2001a) for an early heating of the intergalactic medium which could result in the observed entropy excess. Llyod-Davies \& Ponman (2000) have attempted to determine this extra energy through the binding energy, finding values of about $0.5 \mathrm{keV}$ per proton. Knowing the energy and entropy excess we can actually calculate at which environmental density this entropy excess was created with a result of about $1-210^{-4} \mathrm{~cm}^{-3}$. This gas density is reached in the cosmic mean gas density at redshifts of about 6 to 7 and for an object that is at turn-around and starting to collapse at a redshift of 3 to 4 . The latter is a plausible number for the peak of star formation in a protocluster environment. In addition cooling processes are operative in the group and cluster centers which have to be taken into account.

This rough picture is tested in simulations by a number of authors. Some have shown that the amount of energy needed at the typical time of most of the star formation to produce the entropy excess is much higher than what can be expected from the energy release of supernovae, even if all this energy is dumped into the intracluster medium with negligible spontaneous energy dissipation (e.g. Wu et al. 2000, Borgani et al. 2002). Voit \& Bryan (2000) as well as others (Bryan 2000, Pearce et al. 2000, Muanwong et al. 2001, 2002, Wu \& Xue 2002, Voit et al. 2002 Voit \& Ponman 2003) have shown that the cooling and condensation of the coldest gas in groups and clusters can produce the observational 
effect. But it would imply a much too high degree of conversion of gas into stars (e.g. Balogh et al. 2001). Thus recent models have been more successful by combining the effect of cooling and feedback heating, e.g. by Finoguenov et al. (2003) applying a homogeneous specific energy input of $0.75 \mathrm{kev}$ per particle at a redshift of $z \sim 3$ with additional cooling.

\section{Summary and conclusions}

The above results of XMM-Newton and CHANDRA observations demonstrate that we are starting to gain precise knowledge about the structure of galaxy clusters and the physical state of the ICM. In particular study of the abundance pattern and the entropy structure in the ICM of clusters and groups is shedding light onto the supernova yields, the metal enrichment history in relation to the structural evolution of the cluster, and the star formation evolution. The detailed solutions to most of the questions raised here are still to come. Therefore we will be witnessing a great time for X-ray astronomy providing substantial contributions to the major questions of astrophysics and cosmology.

Acknowledgments. I like to thank my collaborators Kyoko Matsushita, Alexis Finoguenov, Ulrich Briel, Stefano Borgani for the fruitful joint work and the many discussions. I also like to thank the XMM-Newton team at MPE and outside for support and for making these beautiful results possible. XMMNewton, an ESA mission, is funded by ESA Member States and NASA. The XMM-Newton project is supported by the Bundesministerium für Bildung und Forschung, Deutsches Zentrum für Luft und Raumfahrt, The Max-Planck Society and the Haidenhain-Stiftung.

\section{References}

Allen, S.W., Schmidt, R.W., Fabian, A.C., 2002, MNRAS, 334, L11

Balogh, M.L., Pearce, F.R., Bower, R.G. \& Kay, S.T., 2001, MNRAS, 326, 1228

Belsole, E., Sauvageot, J.L., Böhringer, H., et al. , 2000, A\&A, 365, L188

Böhringer, H., Belsole, E., Kennea, J., et al., 2001, A\&A, 365, L18

Böhringer, H., Matsushita, K., Ikebe, Y., et al. 2002, A\&A, 382, 804

Böhringer, H., Matsushita, K., Churazov, E., Finoguenov, A., Chen, Y., 2003, A\&A, 382, 804

Bryan, G.L., 2000, ApJ, 544, L1

Buote, D.A., 1999, MNRAS, 309, 685

De Grandi, S., \& Molendi, S., 2001,

Ettori, S., Tozzi, P., Rosati, P., 2002, A\&A, 398, 879

Ezawa, H., Fukazawa, Y., Makishima, K., et al., 1997, ApJ, 490, L33

Fabian, A.C., 1994, ARA\&A, 32, 277

Finoguenov, A., David, L.P., Ponman, T.J., 2000, ApJ, 514, 188

Finoguenov, A., Arnaud, M., David, L.P., 2001a, ApJ, 555, 191

Finoguenov, A., Reiprich, T.H., Böhringer, H., 2001b, A\&A, 368, 749 
Finoguenov, A., Matsushita, K., Böhringer, H., et al., 2002, A\&A, 381, 21

Finoguenov, A., Borgani, S., Tornatore, L., , Böhringer, H., 2003, A\&A, 398, L35

Fukazawa, Y., Makishima, K., Tamura, T., et al., 1998, PASP, 50, 187

Ivanov, V., Hamuy, M. \& Pinto, P.A., 2000, ApJ, 542, 588

Iwamoto, K., Brachwitz, F., Nomoto, K., et al., 1999, ApJS, 125, 439

Lloyd-Davies, E.J., Ponman, T.J., Cannon, D.B., 2000, MNRAS, 315, 689

Madau, P., Ferguson, H.C., Dickinson, M., et al., 1996, MNRAS, 283, 1388

Matsumoto, H., Koyama, K., Awaki, H., et al., 1996, PASP, 48, 201

Matsushita, K., Belsole, E., Finoguenov, A., Böhringer, H., 2002, A\&A, 386, 77

Matsushita, K., Finoguenov, A., Böhringer, H., 2003a, A\&A,

Matsushita, K., et al. 2003, A\&A submitted

Molendi, S. \& Pizzolato, F., 2001, ApJ, 560, 194

Molendi, S., 2002, ApJ, 580, 815

Muanwong, O., Thomas, P.A., Kay, S.T., et al., 2001, ApJ, 552, L27

Muanwong, O., Thomas, P.A., Kay, S.T., Pearce, F.R., 2002, MNRAS, 336, 527

Navarro, J.F., Frenk, C.S., White, S.D.M., 1997, ApJ, 490, 493

Nomoto, K., Thielemann, F.-K., Yokoi, K., 1984, ApJ, 286, 644

Nomoto, K., Iwamoto, K., Nakasato, N., et al., 1997, Nucl. Phys., A621, 467

Nulsen, P.E.J., 1986, MNRAS, 221, 377

Pearce, F.R., Thomas, P.A., Couchman, H.M.P., Edge, A.C., 2000, MNRAS, 317,1029

Ponman, T.J., Cannon, D.B., Navarro, J.F., 1999, Nature, 397, 135

O'Meara, J.M., Tytler, D., Kirkman, D., et al., 2001, ApJ, 552, 718

Ponman, T.J., Sanderson, A.J.R., Finoguenov, A. 2003, MNRAS, 343, 331

Pratt, G.W. \& Arnaud, M., 2002, A\&A, 394, 375

Reiprich, T.H., 2001, Ph.D. Thesis, Ludwig-Maximilians-Universität München

Reiprich, T.H. \& Böhringer, H., 2002, ApJ, 567, 716

Renzini, A., Ciotti, L., D'Ercole, A., Pellegrini, S., 1993, ApJ, 419, 52

Thomas, P.A., Fabian, A.C., \& Nulsen, P.E.J., 1987, MNRAS, 228, 973

Turner, M.S., 2002, ApJL 576, L101

Voit, M. \& Bryan, G.L., 2001, Nature, 414, 425

Voit, M. \& Bryan, G.L., Balogh, M.L., Bower, R.G., 2002, ApJ, 576, 601

Voit, M. \& Ponman, T.J., 2003, ApJ, 594, L75

White, S.D.M., Navarro, J.F., Evrard, A.E., Frenk, C.S., 1993, Nature, 366, 429

Wu, X.P. \& Xue, Y.-J., 2002, ApJ, 569, 112; ApJ, 572, L19

Woosley, S.E. \& Weaver, T.A., 1995, ApJS, 101, 181 